\title{
Providencia rettgeri infection complicating cranial surgery: illustrative cases
}

\author{
Shabal Sapkota, MD, ${ }^{1}$ Mitesh Karn, ${ }^{2}$ Sanjib Mani Regmi, PhD, ${ }^{3}$ Sushma Thapa, MBBS, ${ }^{1}$ Farhan Uddin Miya, MBBS, ${ }^{1}$ and \\ Sapana Yonghang, MBBS ${ }^{1}$ \\ ${ }^{1}$ Department of Neurosurgery, ${ }^{2}$ School of Medicine, and ${ }^{3}$ Department of Microbiology, Gandaki Medical College Teaching Hospital and Research Center, Pokhara, Nepal
}

BACKGROUND Providencia rettgeri is a rare cause of nosocomial infection in humans. These organisms are capable of biofilm production and are intrinsically resistant to commonly used antibiotics, leading to high rates of morbidity and mortality. P. rettgeri may very rarely cause postneurosurgical infection. OBSERVATIONS In this report, the authors describe two patients in whom P. rettgeri infection complicated the postoperative course. Both the patients underwent craniotomy at approximately the same time under similar environments. The organism isolated was resistant to most of the commonly used antibiotics, and therapy tailored to the results of susceptibility testing led to resolution of infection in both cases.

LESSONS $P$. rettgeri is a rare cause of postneurosurgical nosocomial infection. Timely identification and early tailoring of antibiotic therapy based on susceptibility testing is the key to treatment. Every effort should be made to identify the source of infection and rectify it so that mortality, morbidity, and financial burden are reduced. Contact isolation and use of sterile gloves after each patient contact are effective in preventing its spread, as in most cases of nosocomial infection.

https://thejns.org/doi/abs/10.3171/CASE21318

KEYWORDS infection; neurosurgery; Providencia rettgeri

The genus Providencia is a group of rare, gram-negative, ureaseproducing bacilli from the Enterobacteriaceae family. ${ }^{1}$ Among the species of Providencia currently recognized, Providencia stuartii and Providencia rettgeri are the ones commonly responsible for human infection. $P$. rettgeri is ubiquitous in the environment and is commonly found in water, soil, and animal reservoirs. ${ }^{2}$ In humans, $P$. rettgeri is an opportunistic pathogen associated with nosocomial infection, including catheter-induced urinary tract infection (UTI), bacteremia, skin infections, and gastroenteritis. These organisms are capable of biofilm production and are usually resistant to commonly used antibiotics, leading to high rates of morbidity, mortality, and financial toxicity. ${ }^{3} P$. rettgeri causing infection after neurosurgical procedures is very rare. We describe two cases at a neurosurgical intensive care unit (ICU) in a tertiary care hospital in Nepal where $P$. rettgeri complicated the recovery after cranial surgery.

\section{Illustrative Cases}

\section{Case 1}

A 24-year-oldman presented to us with open, depressed, comminuted fractures of frontal bones and a bifrontal brain contusion after a road traffic accident. A left frontotemporoparietal decompressive craniectomy and frontal contusectomy was carried out. The postoperative period was uneventful until the eighth day after surgery. On the ninth postoperative day, he developed a spiking fever with a maximal recorded temperature of $102^{\circ} \mathrm{F}$. Empirical antibiotics were started on the basis of suspected infection. However, the fever did not subside. Blood counts were significant for leukocytosis; however, culture findings were negative. The patient's chest radiograph was clear. A routine urine examination and culture revealed no abnormalities. Repeat computed tomography of the head showed no signs suggestive of brain abscess. However, the wound drain culture grew $P$. rettgeri sensitive to gentamicin, amikacin, levofloxacin, and imipenem. Because a gentamicin-based regimen had already been tried as a part of empirical therapy without any relief, intravenous levofloxacin was started. Our patient recovered well and was discharged after an additional week of ICU stay.

\section{Case 2}

A 72-year-old hypertensive man presented to us with rightsided hemiparesis. Imaging showed intracerebral hemorrhage with

ABBREVIATIONS ICU = intensive care unit; OR = operating room; UTI = urinary tract infection. INCLUDE WHEN CITING Published August 23, 2021; DOI: 10.3171/CASE21318.

SUBMITTED May 23, 2021. ACCEPTED July 12, 2021.

(c) 2021 The authors, CC BY-NC-ND 4.0 (http://creativecommons.org/licenses/by-nc-nd/4.0/). 
intraventricular extension. He underwent a parietotemporal minicraniotomy with evacuation of a hematoma. A few days after the procedure, our patient developed fever and drowsiness. His chest radiograph was suggestive of pneumonia, and empirical antibiotics were started, without any relief of symptoms. A drain tip culture grew $P$. rettgeri sensitive only to imipenem. Intravenous imipenem was started, and the patient recovered well. He was discharged after 10 days of ICU stay.

\section{Discussion}

The tribe Proteeae of the Enterobacteriaceae family includes three genera: Proteus, Morganella, and Providencia. Providencia is distinct from the other two members in its property to use citrate, ferment D-mannitol, and inability to ferment lactose. ${ }^{4}$ The genus Providencia includes five species: $P$. stuartii, $P$. rettgeri, $P$. alcalifacens, $P$. rustigianii, and $P$. heimbachae. $P$. rettgeri was first isolated in 1904 by Rettger during an epidemic of fowl cholera. It was studied in detail in 1918 by Hadley, and he named it Bacterium rettgeri. In 1943, Rustigian and Stuart assigned this organism to the genus Proteus on the basis of its biochemical properties. It was only in 1978, when Brenner, on the basis of DNA hybridization studies, reassigned Proteus rettgeri to the genus Providencia. ${ }^{5}$

Providencia infection in humans is rare and is usually acquired in a hospital setting. The first description of human infection by $P$. rettgeri dates to 1951, when Goldfarb and De Bakey ${ }^{6}$ reported a case of $P$. rettgeri-associated empyema. Antibiotic-resistant strains were described as early as 1971. P. rettgeri infection in humans is rare and is usually implicated in hospital-acquired UTI and bacteremia. Reports of $P$. rettgeri causing gastroenteritis, wound infection, bacteremia, catheter-associated infections, neonatal sepsis, and bacteremia, among others, have been described. ${ }^{7} P$. rettgeri as a causative agent of neurosurgical infection is rarely described. Maiti et al. $^{8}$ described cases of hospital-acquired $P$. rettgeri meningitis and empyema in which adults with infection died. The main problem of $P$. rettgeri infection is that these organisms are capable of biofilm production and are intrinsically resistant to most of the commonly used antibiotics and therefore have high rates of mortality. Another difficulty in managing such infections is the capability of these organisms to undergo phenotype switching, which leads to rapid evolution of resistant strains during the course of treatment. This shifting pattern of antibiotic susceptibility is a critical issue posed by Providencia-like organisms. $^{9}$

Postoperative infections are a major cause of morbidity and mortality in neurosurgical patients. Gram-positive organisms such as Staphylococcus aureus and polymicrobial flora are usually implicated. Providencia-like organisms are rarely, if ever, implicated as a causative agent of postoperative infection in neurosurgery. In this article, we have described two cases of $P$. rettgeri infection in neurosurgical patients.

\section{Observations}

Identification of the Organism

Organisms were identified using standard protocols of the World Health Organization. In both cases, the organisms isolated were gram-negative; non-lactose fermenting; positive for citrate, urease, and mannitol; and negative for sucrose. Antibiotic susceptibility testing carried out on Mueller Hinton agar using a disk diffusion method recommended by the Clinical and Laboratory Standards Institute guidelines revealed the findings listed in Table 1.
TABLE 1. Antibiotic susceptibility pattern in presented cases

\begin{tabular}{lcc}
\hline Antibiotic Susceptibility & Patient 1 & Patient 2 \\
\hline Imipenem & $\mathrm{S}$ & $\mathrm{S}$ \\
\hline Amikacin & $\mathrm{S}$ & $\mathrm{R}$ \\
\hline Levofloxacin & $\mathrm{S}$ & $\mathrm{R}$ \\
\hline Gentamicin & $\mathrm{S}$ & $\mathrm{R}$ \\
\hline Cefuroxime & $\mathrm{R}$ & $\mathrm{R}$ \\
\hline Ceftriaxone & $\mathrm{R}$ & $\mathrm{R}$ \\
\hline
\end{tabular}

$\mathrm{R}=$ resistant; $\mathrm{S}=$ sensitive.

One interesting observation is that these patients were operated on in the same operating room (OR) and were kept in the same ICU. In both cases, the source of infection could not be identified. Blood and urine cultures were sterile. However, the wound drain in both patients revealed growth of $P$. rettgeri at culture. Biofilm production and colonization of the brain catheters were highly probable. We hypothesize that one of our patients might have been infected from some unknown source in the hospital, and crossinfection occurred between the patients. In the first case, the organism isolated was sensitive to imipenem, amikacin, levofloxacin, and gentamicin. However, in the second patient, the organism was sensitive only to imipenem. Phenotype switching and evolution of antimicrobial resistance are very common with Providencia, and we believe the varying antimicrobial susceptibility seen in our cases is because of this property of such organisms. Antibiotic tailoring based on susceptibility tests resulted in resolution of infection in both the cases. Both of the patients were kept on contact isolation until the end of their admission. After $P$. rettgeri was isolated, the infection control section of our hospital was notified, and the neurosurgery ICU and OR were fumigated. No further cases of $P$. rettgeri have been reported from our neurosurgery ICU to date.

\section{Lessons}

Nosocomial infections are a major cause of postoperative morbidity and mortality in neurosurgery, and $P$. rettgeri is one of the relatively rare causes of postoperative infection after cranial surgery. These organisms are resistant to most of the commonly used antibiotics and carry a high mortality rate. The ease of transfer of antimicrobial resistance genes among these nosocomial pathogens has been recognized as a major threat to public health globally, hence the necessity for timely control of such spread. ${ }^{10}$ Quick identification and early tailoring of antibiotic therapy based on susceptibility testing are the keys to treatment. Every effort should be made to identify the source of these infections and to rectify it. Where available, molecular epidemiological tools should be used to identify the source of infection or cross-infection. In addition to sterilization and equipment cleaning, regular surveillance should be conducted to detect early contamination of equipment and the environment. Most of these infections are seen in immune-compromised patients who undergo invasive procedures such as urinary catheterization, dialysis, and surgical biopsies. Therefore, unnecessary procedures should be strictly limited. Because neurosurgery is highly sensitive, effective and strict infection control measures such as contact precaution, hand hygiene, and regular equipment sterilization should be practiced.

The prevention and control of nosocomial pathogens require a targeted approach addressing universal safety precautions, antimicrobial stewardship, and organism transmission. ${ }^{11}$ Proper and 
hygienic practice often prevents most of these cases of nosocomial infection, including that of $P$. rettgeri. This improves not only patient outcomes but also the economic cost of and patient satisfaction with treatment.

\section{Acknowledgments}

We thank Prof. Dr. Rajeswar Reddy Kasarla, Department of Microbiology, Universal College of Medical Sciences, Bhairahawa, Nepal, for his input on this paper.

\section{References}

1. O'Hara CM, Brenner FW, Miller JM. Classification, identification, and clinical significance of Proteus, Providencia, and Morganella. Clin Microbiol Rev. 2000;13(4):534-546.

2. Wie SH. Clinical significance of Providencia bacteremia or bacteriuria. Korean J Intern Med. 2015;30(2):167-169.

3. Choi HK, Kim YK, Kim HY, Park JE, Uh Y. Clinical and microbiological features of Providencia bacteremia: experience at a tertiary care hospital. Korean J Intern Med. 2015;30(2):219-225.

4. Farmer JJ 3rd, Davis BR, Hickman-Brenner FW, et al. Biochemical identification of new species and biogroups of Enterobacteriaceae isolated from clinical specimens. J Clin Microbiol. 1985;21(1):46-76.

5. Washington MA, Barnhill J, Griffin JM. A case of wound infection with Providencia rettgeri and coincident gout in a patient from Guam. Hawaii J Med Public Health. 2015;74(11):375-377.

6. Goldfarb PM, De Bakey E. Empyema due to Proteus rettgeri: report of a case with recovery. J Med Assoc State Ala. 1951;21(2):33-37.

7. Sharma D, Sharma P, Soni P. First case report of Providencia rettgeri neonatal sepsis. BMC Res Notes. 2017;10(1):536.
8. Maiti TK, Singh VK, Pandey P. Providencia rettgeri: an unusual cause of central nervous system infections. Am J Med Sci. 2013;346(2):158-159.

9. Tada T, Miyoshi-Akiyama T, Dahal RK, et al. NDM-1 metallo- $\beta$-lactamase and ArmA 16S rRNA methylase producing Providencia rettgeri clinical isolates in Nepal. BMC Infect Dis. 2014;14(1):56.

10. Pasteran F, Meo A, Gomez S, et al. Emergence of genetically related NDM-1-producing Providencia rettgeri strains in Argentina. $J$ Glob Antimicrob Resist. 2014;2(4):344-345.

11. Tshisevhe VS, Lekalakala MR, Tshuma N, Janse van Rensburg S, Mbelle N. Outbreak of carbapenem-resistant Providencia rettgeri in a tertiary hospital. S Afr Med J. 2016;107(1):31-33.

\section{Disclosures}

The authors report no conflict of interest concerning the materials or methods used in this study or the findings specified in this paper.

\section{Author Contributions}

Conception and design: Sapkota, Karn, Regmi, Miya. Acquisition of data: Sapkota, Karn, Regmi, Thapa, Yonghang. Analysis and interpretation of data: Sapkota, Miya, Yonghang. Drafting the article: Sapkota, Karn, Miya, Yonghang. Critically revising the article: Sapkota, Karn, Regmi, Miya, Yonghang. Reviewed submitted version of manuscript: Karn, Regmi, Miya. Administrative/technical/material support: Karn, Miya, Yonghang. Study supervision: Regmi.

\section{Correspondence}

Shabal Sapkota: Gandaki Medical College Teaching Hospital and Research Center, Pokhara, Nepal. shabalsapkota@gmail.com. 